\title{
Comparison of Suction Catheter versus Forceps Biopsy for Sampling of Solitary Pulmonary Nodules Guided by Electromagnetic Navigational Bronchoscopy
}

\author{
Ralf Eberhardt $^{a}$ Ross K. Morgan ${ }^{b} \quad$ Armin Ernst $^{b} \quad$ Thomas Beyer $^{c}$ \\ Felix J.F. Herth ${ }^{a}$ \\ a Department of Pneumology and Critical Care Medicine, Thoraxklinik, University of Heidelberg gGmbH, \\ Heidelberg, Germany; ${ }^{b}$ Interventional Pulmonology, Beth Israel Deaconess Medical Center, Harvard \\ Medical School, Boston, Mass., USA; ' $D$ epartment of Pulmonology, Lungenklinik Ballenstedt/Harz gGmbH, \\ Ballenstedt, Germany
}

\section{Key Words}

Bronchoscopy - Catheter aspiration - Electromagnetic navigation · Endobronchial ultrasound · Lung cancer • Pulmonary nodules $\cdot$ Transbronchial biopsy

\begin{abstract}
Background: Electromagnetic navigation has been approved for use as an adjunct to standard bronchoscopy. The diagnostic yield varies depending on the size of the lesion and successful navigation to the lesion. Objectives: The performance of two different biopsy tools, i.e. catheter aspiration and forceps biopsy, in the diagnosis of small pulmonary nodules (SPN) guided by electromagnetic navigational bronchoscopy (ENB) was examined. Methods: 54 patients referred for suspected lung cancer underwent ENB and 55 SPN $(<3 \mathrm{~cm})$ were sampled using both techniques. Endobronchial ultrasound (EBUS) was used to verify the accuracy of target lesion localization by ENB. Primary end points of the study were successful navigation to the lesion and a positive diagnosis. Patients were followed until a definitive diagnosis was obtained. Results: All 55 lesions were accessed. Two lesions were excluded from data analysis as the patients were lost to follow-up and their diagnoses could not be confirmed. Of
\end{abstract}

the remaining 53 lesions, 40 samples (75.5\%) were diagnostic. Compared to forceps biopsy, catheter aspiration was positively correlated with the success rate (36/40 vs. 22/40; $p=0.035)$. The diagnostic yield was $93 \%$ when EBUS verified the lesion location after navigation and only $48 \%$ when lesion location was not confirmed. There were no significant complications. Conclusions: ENB is a useful tool in the evaluation of SPN $<3 \mathrm{~cm}$ in diameter. For malignant lesions, sampling by catheter aspiration is associated with a higher diagnostic yield than sampling by forceps biopsy alone, in particular when EBUS could not confirm lesion location prior to sampling.

Copyright $\odot 2009$ S. Karger AG, Basel

\section{Introduction}

Radiologically, a solitary pulmonary nodule (SPN) is defined as an intraparenchymal lung lesion $<3 \mathrm{~cm}$ in diameter not associated with atelectasis or adenopathy [1]. The likelihood of malignancy for SPNs between 0.8 and $2.0 \mathrm{~cm}$ was previously reported to be about $18 \%$, being $\sim 50 \%$ for nodules $>2.0 \mathrm{~cm}[2,3]$. One of the diagnostic challenges remains the difficulty in reaching these small

\section{KARGER}

Fax +41613061234 E-Mail karger@karger.ch www.karger.com (c) 2009 S. Karger AG, Basel

0025-7931/10/0791-0054\$26.00/0

Accessible online at:

www.karger.com/res
Dr. med. Ralf Eberhardt

Thoraxklinik am Universitätsklinikum Heidelberg gGmbH

Amalienstrasse 5, DE-69126 Heidelberg (Germany)

Tel. +496221 396 1201, Fax +496221 3961205

E-Mail ralf.eberhardt@thoraxklinik-heidelberg.de 
lung lesions and successfully obtaining adequate tissue samples for pathological diagnosis.

Standard flexible bronchoscopy is limited in its ability to evaluate small pulmonary nodules [4]. In the absence of visible endobronchial abnormality, its yield varies from 20 to $84 \%$ in malignant lesions, and from 35 to $56 \%$ in benign ones [5-8]. For peripheral lesions $<2 \mathrm{~cm}$, the yield of bronchoscopy can be as low as $14 \%$ [5]. As a result, suspicious peripheral small lung lesions are often biopsied with more invasive techniques, such as CT-guided fine needle aspiration (with an increased risk of pneumothorax) or surgical biopsy via video-assisted thoracoscopic surgery or thoracotomy.

Electromagnetic guidance allows the bronchoscopist to steer endoscopic tools to targeted lung lesions. The diagnostic yield of electromagnetic navigation (EN)-guided bronchoscopy (ENB) has been as high as $74.1 \%$ in a number of settings [9-13]. To date, there have been no reports on the utility of this system solely for the diagnosis of SPNs $(<3 \mathrm{~cm})$ or on the use of alternative sampling techniques, e.g. catheter aspiration, which may result in superior yields than biopsy [14].

The objective of this study was to assess the efficiency of ENB and to compare forceps biopsy with catheter aspiration as tissue sampling techniques in the diagnosis of small peripheral lung lesions $<3 \mathrm{~cm}$ in diameter. Endobronchial ultrasound (EBUS), known to be a safe and effective imaging tool for transbronchial biopsy [13, 15-17], was used prior to sampling for the purpose of lesion verification.

\section{Materials and Methods}

This prospective single-center study was approved by the Ethics Committee of the University of Heidelberg. The patients included were those referred for evaluation of small peripheral pulmonary lesions where there was a suspicion of lung cancer. Fiftyfour patients were included between September 2005 and July 2006 after signing an Ethics Committee-approved informed consent. One SPN was biopsied in every patient with the exception of 1 patient who had 2 lesions biopsied. All procedures were performed under general anesthesia.

\section{Procedure}

All patients had non-contrast CT scans of the chest with a slice thickness of 2-3.5 mm and a slice interval (with overlap of $1 \mathrm{~mm}$ ) of 1-2.5 mm. Basic ENB was performed in a standard fashion. Registration points were defined by identifying 5-9 prominent anatomical landmarks and the location of the target lesion on the virtual bronchoscopy images. An adult-size therapeutic bronchoscope (IT 160, Olympus, Tokyo, Japan) with a 2.8 -mm working channel was used. The sensor probe, which has a width of $1.9 \mathrm{~mm}$

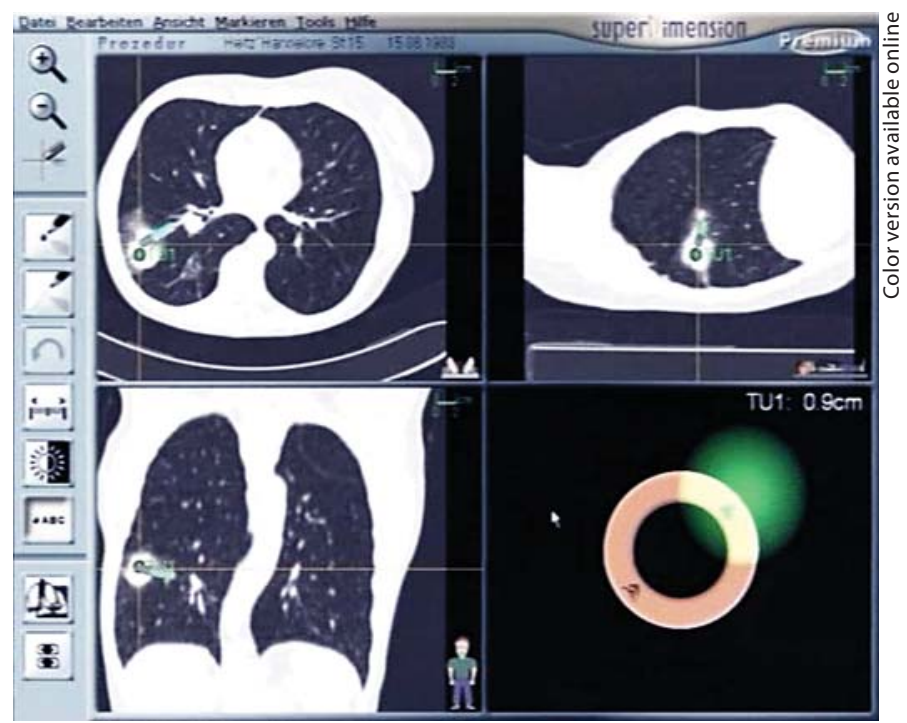

Fig. 1. Typical screen of superDimension ${ }^{\mathrm{TM}}$ software during the ENB.

and a working length of $972 \mathrm{~mm}$, was deployed to the target lesion through an extended working channel (EWC).

A registration error was calculated by the computer software representing the radius of the expected difference between the tip of the sensor probe in the actual patient and its expected location. By either repositioning a misplaced landmark or by eliminating the landmarks with the largest deviation, registration error could be optimized. Navigation was considered complete when the bronchoscope was wedged in the suspected bronchial segment and the sensor probe within the EWC was successfully steered to the lesion based on the multiplanar CT images and the 'tip-view' orientation guide (fig. 1). Navigation error was defined as the discrepancy in the distance between the sensor probe and the lesion.

After completing navigation to the lesion, EBUS was used to verify the relative position of the EN-steerable sensor and the lesion. However, no additional navigation was performed based on the EBUS images, which were used solely for subsequent data analysis and comparison of performance characteristics of two different biopsy techniques. Specifically, specimens were obtained via EWC by:

- catheter suction (Olympus Cannula PR-2B-1 2.0 mm in diameter) and

- standard biopsy forceps (Olympus FB-19-C $120 \mathrm{~cm}$ long).

For each patient, two cytological samples were obtained by catheter aspiration followed by five biopsies with the forceps. The catheter aspiration technique included moving the catheter back and forth while continued suction was applied with a $10-\mathrm{ml}$ syringe. Cytological specimens were smeared on glass slides and air-dried before sending them to an independent cytological laboratory (Lungenklinik Ballenstedt/Harz; fig. 2). Prior to transbronchial biopsy, the EWC position was reassessed by EN and repositioned if necessary. The histological examination of these 


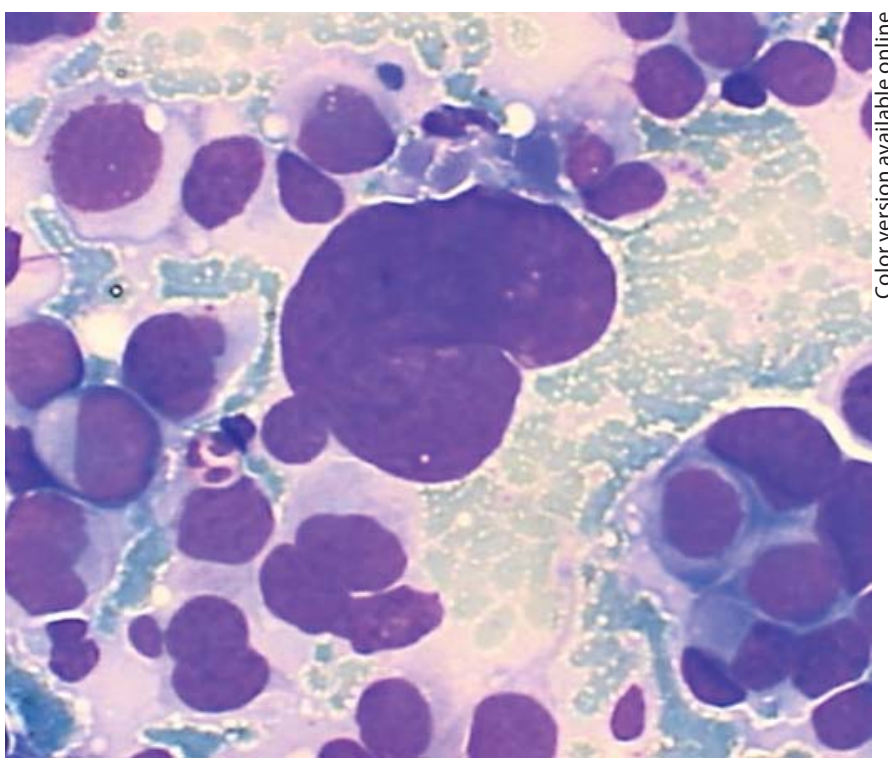

Fig. 2. Aspiration cytology with malignant cell complexes consistent with non-small cell lung cancer (atypical giant nucleus in the center of the slide).

specimens was performed without the knowledge of the cytology results in the Department of Pathology at the University of Heidelberg.

For this study, fluoroscopic imaging and rapid on-site evaluation of biopsy samples were not applied. In all patients, a chest $\mathrm{X}$-ray was performed after the procedure to evaluate iatrogenic pneumothorax after transbronchial lung biopsy. Overall data related to EN system performance, safety and diagnostic yield were recorded. Study parameters, e.g. procedure time, distance to the targeted lesion and success rates, as well as lesion parameters, e.g. size and locations, were recorded and analyzed.

All patients were followed up until either a definitive diagnosis was obtained or the diagnosis was verified by other standard techniques, such as CT-guided fine needle aspiration or surgery.

\section{Statistics}

Descriptive statistics for all continuous variables were summarized as means, medians and standard deviations. Statistical analyses were performed using commercially available software (SPSS, version 14.0) at the Statistical Laboratory, Department of Statistics and Operation Research, School of Mathematical Sciences, Tel Aviv University, Tel Aviv, Israel.

A number of parameters were evaluated, including patient demographics, lesion size and location, distance from the tip of the steerable probe to the center of the peripheral lesion, registration accuracy, diagnostic yield by patients and by biopsy methods, and procedural complication rates. In order to achieve normal distributions, logarithmic transformation was applied to the continuous variables such as duration (registration, navigation and total bronchoscopy time), size and distance to the target center. A t test was used to analyze the relationship between the aforementioned parameters and diagnostic yield (success/failure of ENB), a $\chi^{2}$ test was applied for categorical parameters, and the McNemar test to compare the differences in success rates between the two biopsy methods (catheter aspiration and forceps biopsy). For statistical tests of association, $\mathrm{p} \leq 0.05$ was considered significant.

\section{Results}

ENB was performed on all 54 patients enrolled (14 women and $40 \mathrm{men}$ ), and 55 lesions were identified, successfully accessed and sampled for diagnosis. Two patients were excluded from the diagnostic yield analysis because their benign diagnoses obtained during the ENB procedure could not be further verified (1 patient died of unrelated causes and 1 refused further evaluation).

\section{Patient Demographics, and Lesion and Procedure \\ Characteristics}

The mean age of the patients was 65.1 years (range 29-84 years). The majority of the patients (78.8\%) presented with a smoking history and $36.5 \%$ of them were active smokers. Twelve patients had a history of non-pulmonary cancer. Thirty-two patients had additional pulmonary diseases, such as chronic obstructive pulmonary disease (27 patients) and pneumonia (4 patients). The size of the lesions ranged from 11 to $29 \mathrm{~mm}$, with a mean of $23.3 \mathrm{~mm}$ (long axis). All lesions were sampled twice by catheter suction and five times by forceps except for 1 lesion that was sampled by catheter suction only. The navigation error for this lesion was $>20 \mathrm{~mm}$, and the lesion was not investigated by forceps biopsy due to the high risk of complications. Fifty-six percent of the lesions were located in the left lung and 60\% were located in the upper lobes.

The total bronchoscopy procedure time ranged from 16 to $45 \mathrm{~min}$, with a mean duration of $25.7 \mathrm{~min}$. Mean registration time lasted $2.9 \mathrm{~min}$ (range 1-9 min). Mean navigation time was $3.5 \mathrm{~min}$ (range $0.3-14 \mathrm{~min}$ ).

The mean average fiducial target registration error (AFTRE), a marker used to describe the accuracy of anatomical registration and endobronchial mapping, denotes the divergence of the location between CT and anatomical targets. In this study, AFTRE was $3.6 \mathrm{~mm}$, ranging from 1.8 to $5.7 \mathrm{~mm}$. In 16 cases, registration results were corrected by additional navigation. The mean AFTRE value of these 16 cases improved from 4.9 to 3.6 $\mathrm{mm}$ after the correction. The mean distance from the steerable probe to the center of the targeted peripheral lesion was $0.9 \mathrm{~cm}$ (range $0.2-2.3 \mathrm{~cm}$; table 1 ). 
Table 1. ENB parameters

\begin{tabular}{lr}
\hline Bronchoscopy duration, min & $25.7 \pm 5.8$ \\
Registration duration, min & $2.9 \pm 1.6$ \\
Navigation duration, min & $3.5 \pm 2.8$ \\
AFTRE value, mm & $3.6 \pm 0.9$ \\
Distance to the lesion, $\mathrm{mm}$ & $9 \pm 5.0$ \\
Lesion size, $\mathrm{mm}$ & $23.3 \pm 4.4$ \\
\hline
\end{tabular}

Table 2. Established diagnoses in the study patients

\begin{tabular}{lrcc}
\hline Diagnoses & All & ENB success & Yield, \% \\
\hline Malignancy & 47 & 34 & 72.3 \\
$\quad$ NSCLC & 42 & 31 & 73.8 \\
$\quad$ SCLC & 2 & 1 & 50.0 \\
$\quad$ Metastasis & 3 & 2 & 66.7 \\
Pulmonary abscess & 2 & 2 & 100.0 \\
Tuberculosis & 2 & 2 & 100.0 \\
Benign inflammation & 1 & 1 & 100.0 \\
Scar tissue & 1 & 1 & 75.5 \\
\hline All & 53 & 40 & \\
Dropout & 2 & &
\end{tabular}

NSCLC $=$ Non-small cell lung cancer; SCLC $=$ small cell lung cancer.

\section{Diagnostic Yield}

Of the 53 cases included in the analysis, a definitive cytohistological diagnosis was made on 40 lesions $(75.5 \%)$ after ENB, of which 34 were malignant (table 2). Of the remaining 13 lesions, in 4 tumor was suspected after cytopathological examination, and 9 were considered nondiagnostic. Of the 13 cases, 12 underwent further diagnostic testing and all were found to be malignant; 5 after CT-guided biopsy, 1 following a repeat bronchoscopy and 4 at surgery. In the case of suspected tumor not subjected to further testing, the nodule grew over time and was considered malignant, although the patient refused pathological confirmation. All of these 13 cases were considered ENB failures. Overall, 47 of the 53 lesions were malignant, and the sensitivity and specificity of ENB in the diagnosis of malignancies were calculated to be 72.3 and $100.0 \%$, respectively. There was no statistically significant difference comparing the yield of the biopsies with the location of the nodules in the lung.

ENB to Diagnose Small Lung Lesions
Six of the 40 lesions diagnosed after ENB were not malignant. Two cases were found to have tuberculosis and 2 patients had a lung abscess. In 1 patient, inflammatory changes were only seen on biopsy and the lesion subsequently disappeared following antibiotic treatment, and in a further case, in which the biopsy suggested fibrosis and cytology was negative, the nodule remained stable during the subsequent 2-year follow-up and was felt to represent a scar.

\section{Diagnostic Yield - Aspiration versus Biopsy}

Overall, sampling using the catheter suction technique was found to have a significantly higher diagnostic yield than using forceps ( $\mathrm{p}=0.035)$. Of the 40 cases diagnosed using ENB, in 36 (90\%), the diagnosis was made using catheter suction, but in only 22 cases using forceps (55\%). In 18 of the 40 (45.0\%) lesions, only the specimens obtained from catheter suction were positive, whereas forceps biopsy alone yielded the definitive histological diagnoses in only $4(10.0 \%)$ lesions. In the remaining 18 (45.0\%) lesions, both sampling methods obtained definitive pathological diagnoses.

\section{Lesion Verification Using EBUS}

After navigational bronchoscopy and prior to sampling, 30 of the 55 lesions (54.5\%) were visualized by the EBUS probe inserted via the EWC. Of these 30, 28 (93\%) had diagnostic findings on pathology after either aspiration ( 24 of 28 ) or biopsy (18 of 28). Of the remaining 25 lesions where lesion location following ENB could not be verified by EBUS prior to sampling, only $12(48 \%)$ were diagnosed after sampling. All of these 12 were identified cytologically with the help of the aspiration technique and only 4 were positive on forceps biopsy (fig. 3).

\section{Safety}

One pneumothorax $(1.9 \%)$ was detected by chest Xray after the procedure, but its extent was small and no intervention was deemed necessary. Two patients died during the follow-up period: one died of respiratory failure after surgery and the other as a result of B-cell lymphoma of the colon. No ENB-related adverse events were recorded.

\section{Discussion}

There is an increasing demand to provide alternatives to surgical procedures in the diagnosis of peripheral lung tumors. Many patients are unable to tolerate these proce- 
Fig. 3. Overview of ENB results according to EBUS verification of the target.

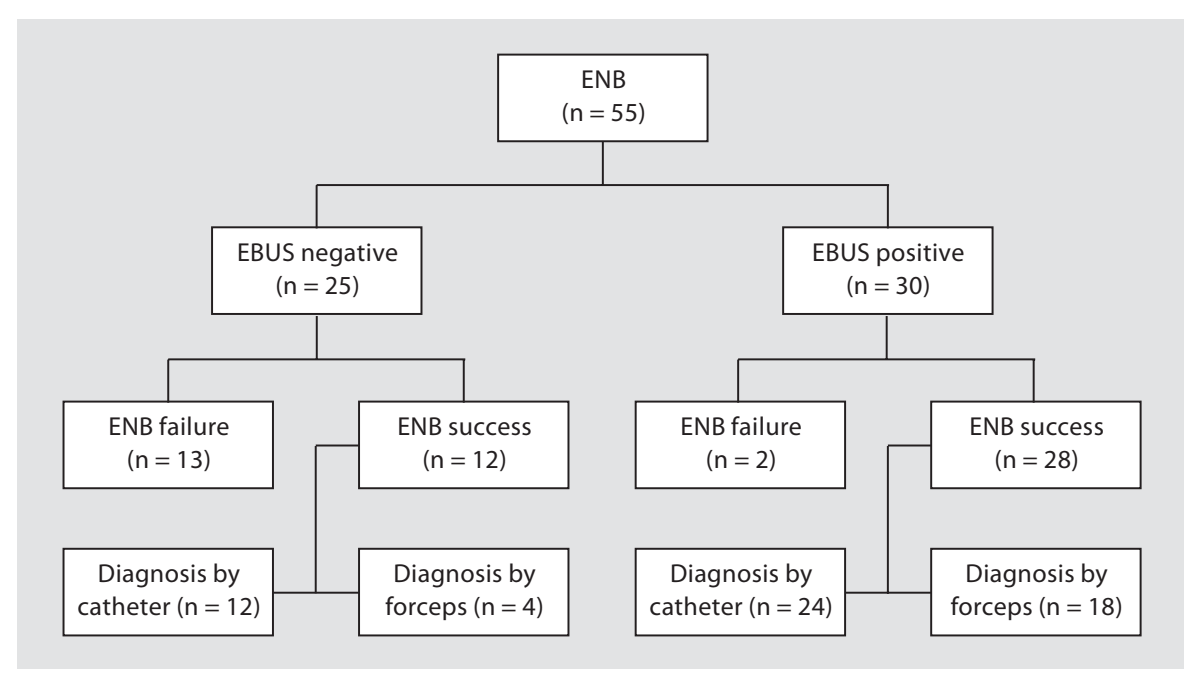

dures because of comorbidities such as impaired lung capacity or heart disease. Transthoracic biopsy by CT guidance is associated with exposure to radiation and a significant risk of pneumothorax.

In our study, the overall diagnostic yield from ENB was $75.5 \%$ and this was independent of lesion size, lesion location or technical parameters, including registration error or navigation precision, being consistent with previously reported experiences using the ENB method with and without fluoroscopy $[9,11,13]$. The mean AFTRE (registration error) was similar to that published in a recent study which described the dependence of the ENB diagnostic yield on this value [10]. While there was no correlation between registration error and yield in our study, our mean AFTRE value was relatively low $(3.6 \mathrm{~mm})$, and this may have improved navigation accuracy. The distance to the target (navigation error) was similar to previous reports [9-11]. The contribution of these factors to the success of ENB remains to be proven. The overall bronchoscopy time was short compared to early publications using the ENB technique [11] and comparable to our recent publications $[9,13]$, which may be attributable to the experience gained from the repeated use of ENB. The registration time is the only actual additional time to standard bronchoscopy and remained very low with a mean of $2.9 \mathrm{~min}$, in agreement with previous publications $[9,13]$. Elimination of imaging time such as fluoroscopy probably decreases the overall procedure duration marginally. Moreover, it also avoids fluoroscopy-induced radiation exposure to the patients and medical staff.

The sensitivity of transbronchial needle aspiration (TBNA) in diagnosing peripheral lung lesions is greater than that of transbronchial biopsy alone [18-20]. There are no published data on the use of TBNA needles in the ENB setting. We hypothesized that catheter aspiration for sampling peripheral lesions would improve the overall diagnostic yield of ENB. The softness of the catheter facilitates its introduction into the EWC with a low risk of complications such as pneumothorax or perforation of the working channel. In addition, and in contrast to the TBNA needle, the soft catheter would not be expected to dislodge the working channel. This study found that catheter aspiration combined with forceps biopsy improves the diagnostic yield after ENB for small peripheral lesions. Overall, 40 of the 53 cases had diagnostic findings; in 18 cases only cytological specimens obtained by aspiration were positive, in a further 18 both cytology and biopsy specimens were positive and in 4 only the biopsy was diagnostic.

The expected rate of malignancy in lesions $>20 \mathrm{~mm}$ exceeds $50 \%$ but varies widely depending on the population studied $[2,3]$. There was a high rate of malignancy in our study population; however, this was not unexpected as most patients are referred with a high index of suspicion for malignancy to our center. All of the benign cases in this study were identified by ENB, but given the small number of benign findings ( 6 cases), we could not draw any definite conclusion about the ENB sampling yield in this setting.

Our group has recently shown that the combination of EBUS and ENB results in a higher diagnostic yield than either method alone [13]. In the present study, we found that lesion verification by radial probe EBUS confirmed the EWC to be located at or within the lesion in $55 \%$ of 
cases. When EBUS confirmed the lesion location, tissue sampling was successful in making a diagnosis in $60 \%$ of cases using forceps biopsy and in $80 \%$ of cases by the aspiration method. In EBUS-negative cases, cytological specimens obtained from catheter aspiration were positive in about half of the cases (12 of 25), whereas forceps biopsy performed poorly (4 of 25). We suspect that the suction method may be useful in cases in whom the lesion was not 'exactly' reached, possibly because the catheter is moved in and out with suction applied, thus increasing diagnosis. We did not use fluoroscopy, but it is possible that fluoroscopy would have improved our yield with the forceps biopsy.

The catheter aspiration tool is not widely used in bronchoscopy, although the technique is similar to TBNA, a technique familiar to many bronchoscopists. In addition, cytology performance can vary widely among laboratories. While this may limit the generalizability of our findings, we believe additional studies on this technique are warranted. Although our study patients were examined under general anesthesia, our preferred approach to these procedures, it has previously been shown that ENB can also be safely and effectively performed with moderate sedation.

ENB combined with radial EBUS is a useful, minimally invasive tool with diagnostic yields for small peripheral lung tumors approaching those achieved with the traditional transthoracic or surgical techniques [2125]. The procedure appears safe. Catheter aspiration performs very well for SPN diagnosis via this route and should be used in addition to biopsy to increase the diagnostic yield. Obviously, performing bronchoscopy with the help of EN and EBUS is expensive. The EN equipment costs roughly 90,000-100,000 EUR and there are disposables to consider, e.g. catheters and locatable guides at $\sim 700-1,000$ EUR/case - all this in the face of lacking reimbursement. However, the aim of this study was to compare the results obtained using two diagnostic tools and not their different costs. However, in order to assess cost efficiency and relative effectiveness, the performance characteristics of the tests have to be assessed. Further studies including this issue are required to make recommendations regarding the preferred use of biopsy techniques and to assess if there is a role for bronchoscopic biopsy in the diagnosis of peripheral lesions.

\section{Acknowledgments}

The locatable sensor probes for ENB were provided free of charge by superDimension (Herzliya, Israel). Analyses were performed by the Statistical Laboratory, Department of Statistics and Operation Research, School of Mathematical Sciences, Tel Aviv University, Tel Aviv, Israel.

\section{References}

1 Tuddenham WI: Glossary of terms for thoracic radiology: recommendations of the Nomenclature Committee of the Fleischner Society. Am J Roentgenol 1984;43:509-517.

$\checkmark 2$ LeefJL 3rd, Klein IS: The solitary pulmonary nodule. Radiol Clin North Am 2002;40:123143.

-3 Midthun DE, Swensen SJ, Jett JR, Hartman TE: Evaluation of nodules detected by screening for lung cancer with low dose spiral computed tomography. Lung Cancer 2003;41 (suppl 2):S40.

4 Libby DM, Smith JP, Altorki NK, Pasmantier MW, Yankelevitz D, Henschke CI: Managing the small pulmonary nodule discovered by CT. Chest 2004;125:1522-1529.

5 Baaklini WA, Reinoso MA, Gorin AB, Sharafkaneh A, Manian P: Diagnostic yield of fiberoptic bronchoscopy in evaluating solitary pulmonary nodules. Chest 2000;117: 1049-1054.

\footnotetext{
6 Shiner RJ, Rosenman J, Kaz I, Reichart N, 11 Gildea TR, Mazzone PJ, Karnak D, Meziane Hershko E, Yellin A: Bronchoscopic evaluation of peripheral lung tumours. Thorax 1988:43:887-889.

7 Chechani V: Bronchoscopic diagnosis of solitary pulmonary nodules and lung masses in the absence of endobronchial abnormality. Chest 1996;109:620-625.

8 Mori K, Yanase N, Kaneko M, Ono R, Ikeda $\mathrm{S}$ : Diagnosis of peripheral lung cancer in cases of tumors $2 \mathrm{~cm}$ or less in size. Chest 1989; 95:304-308.

-9 Eberhardt R, Anantham D, Herth F, FellerKopman D, Ernst A: Electromagnetic navigation diagnostic bronchoscopy in peripheral lung lesions. Chest 2007;131:18001805.

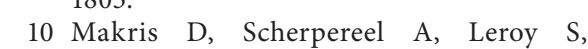
Bouchindhomme B, Faivre JB, Remy J, Ramon P, Marquette CH: Electromagnetic navigation diagnostic bronchoscopy for small peripheral lung lesions. Eur Respir J 2007;29:472-478.

M, Mehta A: Electromagnetic navigation diagnostic bronchoscopy: a prospective study. Am J Respir Crit Care Med 2006;174:982989.

12 Eberhardt R, Anantham D, Ernst A, FellerKopman D, Herth F: Multimodality bronchoscopic diagnosis of peripheral lung lesions: a randomized controlled trial. Am J Respir Crit Care Med 2007;176:36-41.

13 Schwarz Y, Greif j, Becker H, Ernst A, Mehta A: Real-time electromagnetic navigation bronchoscopy to peripheral lung lesions using overlaid CT images: the first human study. Chest 2006;129:988-994.

14 Franke KJ, Nilius G, Rühle KH: Transbronchiale Biopsie im Vergleich zur Katheteraspiration bei peripheren Lungenherden. Pneumologie 2006;60:7-10.

15 Herth FJF, Eberhardt R, Becker HD, Ernst A: Endobronchial ultrasound-guided transbronchial lung biopsy in fluoroscopically invisible solitary pulmonary nodules: a prospective trial. Chest 2006;129:147-150.
} 
16 Yang MC, Liu WT, Lin HC, Lin HC, Chen HC, Chou CL, Hsueh S, Kuo HP: Diagnostic value of endobronchial ultrasound-guided transbronchial lung biopsy in peripheral lung cancers. J Formos Med Assoc 2004; 103: $116-129$.

-17 Shirakawa T, Imamura F, Hamamoto J, Honda I, Fukushima K, Sugimoto M, Shirkakusa T: Usefulness of endobronchial ultrasonography for transbronchial lung biopsies of peripheral lung lesions. Respiration 2004;71: 260-268.

18 Gasparini S, Ferretti M, Secchi EB, Baldelli S, Zuccatosta L, Gusella P: Integration of transbronchial and percutaneous approach in the diagnosis of peripheral pulmonary nodules or masses: experience with 1,027 consecutive cases. Chest 1995;108:131-137.
19 Tan BB, Flaherty KR, Kazerooni EA, Iannettoni MD: The solitary pulmonary nodule. Chest 2003;123(suppl 1):89-96.

20 Reichenberger F, Weber J, Tamm M, Bolliger CT, Dalquen P, Perruchoud AP, Soler M: The value of transbronchial needle aspiration in the diagnosis of peripheral pulmonary lesions. Chest 1999;116:704-708.

-21 Schreiber G, McCrory DC: Performance characteristics of different modalities for diagnosis of suspected lung cancer: summary of published evidence. Chest 2003;123(1 suppl):115S-128S.

22 DeCamp MM Jr, Jaklitsch MT, Mentzer SJ, Harpole DH Jr, Sugarbaker DJ: The safety and versatility of video-thoracoscopy: a prospective analysis of 895 consecutive cases. J Am Coll Surg 1995;181:113-120.
23 Geraghty PR, Kee ST, McFarlane G, Razavi MK, Sze DY, Dake MD: CT-guided transthoracic needle aspiration biopsy of pulmonary nodules: needle size and pneumothorax rate. Radiology 2003;229:475-481.

24 Yeow KM, Su IH, Pan KT, Tsay PK, Lui KW, Cheung YC, Chou AS: Risk factors of pneumothorax and bleeding: multivariate analysis of 660 CT-guided coaxial cutting needle lung biopsies. Chest 2004;126:748-754.

25 Allen MS, Deschamps C, Jones DM, Trastek VF, Pairolero PC: Video-assisted thoracic surgical procedures: the Mayo experience. Mayo Clin Proc 1996;71:351-359. 